\title{
A TRUNCATION METHOD FOR DETECTING SINGULAR MINIMIZERS INVOLVING THE LAVRENTIEV PHENOMENON
}

\author{
YU BAI \& ZHIPING $\mathrm{LI}^{\dagger}$ \\ LMAM \& SCHOOL OF MATHEMATICAL SCIENCES, \\ PEKING UNIVERSITY, BEIJING 100871, P.R.CHINA
}

\begin{abstract}
A numerical method using the truncation technique on the integrand is developed for computing singular minimizers or singular minimizing sequences in variational problems involving the Lavrentiev phenomenon. It is proved that the method can detect absolute minimizers with various singularities whether the Lavrentiev phenomenon is involved or not. It is also proved that, when the absolute infimum is not attainable, the method can produce minimizing sequences. Numerical results on the Manià's example and a 2-dimensional problem involving the Lavrentiev phenomenon with continuous Sobolev exponent dependence, are given to show the efficiency of the method.
\end{abstract}

\section{INTRODUCTION}

Singular minimizers are commonly seen in variational problems, and some of the singular minimizers can be tricky to compute. For instance, consider the well known example, given by Manià [17], of minimizing the integral functional

$$
I(u)=\int_{0}^{1}\left(u^{3}-x\right)^{2}\left(u^{\prime}\right)^{6} d x
$$

in the set of admissible functions

$$
\mathcal{A}^{1}=\left\{u \in W^{1,1}(0,1): u(0)=0, u(1)=1\right\} .
$$

1991 Mathematics Subject Classification. 65K10,65N30,49A10.

Key words and phrases. truncation method, singular minimizer, Lavrentiev phenomenon, minimizing sequence, convergence.

The research was supported in part by the Special Funds for Major State Basic Research Projects (G1999032804), NSFC (10431050) and RFDP of China.

$\dagger$ Corresponding author: Zhiping Li, email address: lizp@math.pku.edu.cn. 
It is easy to verify that $\hat{u}=x^{\frac{1}{3}} \in \mathcal{A}^{1}$ is the unique minimizer of $I(\cdot)$ in $\mathcal{A}^{1}$, and that $I(\hat{u})=0$. To compute such a singular minimizer, the most obvious initial approach is to use standard finite element methods. But the facts that

$$
\inf _{u \in \mathcal{A}^{1} \cap W^{1, \infty}(0,1)} I(u)>\inf _{u \in \mathcal{A}^{1}} I(u)=0,
$$

which is known as the Lavrentiev phenomenon, first found by Lavrentiev in 1926 [12], and that

$$
\begin{array}{r}
\lim _{j \rightarrow+\infty} I\left(u_{j}\right)=+\infty, \quad \text { if } u_{j} \in \mathcal{A}^{1} \cap W^{1, p}(0,1), \text { for some } p \geqslant \frac{3}{2}, \\
\text { and } u_{j} \rightarrow \hat{u} \text { a.e. } x \in(0,1),
\end{array}
$$

which is proved by Ball and Mizel [3], show that typically such approaches can neither detect the minimizer nor determine the infimum.

In general, for the problem of minimizing an integral functional

$$
I(u)=\int_{\Omega} f(x, u(x), D u(x)) d x
$$

in a set of admissible functions

$$
\mathcal{A}^{p}=\left\{u \in W^{1, p}\left(\Omega ; R^{m}\right): u=u_{0}, x \in \partial \Omega_{0}\right\}
$$

for some given $1 \leqslant p \leqslant+\infty$, where $\Omega \subset R^{n}$ is a bounded open set with Lipschitz continuous boundary $\partial \Omega$ and $\partial \Omega_{0} \subset \partial \Omega$ with meas $_{n-1}\left(\partial \Omega_{0}\right) \neq 0$, and where $f: \Omega \times R^{m} \times R^{m n} \rightarrow R$ is a given real function, there are four possible cases:

(case 1): The problem does not involve the Lavrentiev phenomenon, i.e.

$$
\inf _{u \in \mathcal{A}^{1}} I(u)=\inf _{u \in \mathcal{A}^{\infty}} I(u),
$$

and the infimum of the problem is attainable for some $p \geqslant 1$;

(case 2): The problem does not involve the Lavrentiev phenomenon and the infimum of the problem is not attainable for any $p \geqslant 1$;

(case 3): The problem involves the Lavrentiev phenomenon, i.e. there exist $1 \leqslant q<r \leqslant+\infty$ such that

$$
\inf _{u \in \mathcal{A}^{1}} I(u)=\inf _{u \in \mathcal{A}^{q}} I(u)<\inf _{u \in \mathcal{A}^{r}} I(u)=\inf _{u \in \mathcal{A}^{\infty}} I(u),
$$

and the infimum of $I(\cdot)$ in $\mathcal{A}^{p}$ is attainable for a given $p \geqslant 1$; 
(case 4): The problem involves the Lavrentiev phenomenon and the infimum of $I(\cdot)$ in $\mathcal{A}^{p}$ is not attainable for a given $p \geqslant 1$.

Various numerical methods for detecting singular minimizers involving the Lavrentiev phenomenon have been developed in recent years $[4,13,14,15]$ (see [5] for a survey and more references), and corresponding convergence theorems were proved to guarantee that these methods can be successfully used in detecting singular minimizers in case 3 when $1<p \leqslant q$ (see (1.8)).

In the present paper, we designed a new truncation method and proved some convergence theorems, which not only enable us to apply the truncation method to all the four cases for all $1 \leqslant p \leqslant+\infty$, but also enable us to develop some more practical techniques to determine the truncation regions and parameters in the process of applying the truncation method to computing singular minimizers, or minimizing sequences when the infimum is unattainable, which may involve the Lavrentiev phenomenon.

The rest of the paper is organized as follows. A lower semicontinuity theorem [16], which is useful in the convergence analysis of the method, is given in Section 2. In Section 3, we establish the new truncation method and the convergence theorems. In Section 4, the numerical results on the Manià's example and a 2-dimensional problem with continuous Sobolev exponent dependence given by Foss [10] are presented to show the efficiency of our method.

\section{A LOWER SEMICONTINUITY THEOREM}

We first introduce some definitions. Let $\Omega \subset R^{n}$ be bounded and open.

Definition 2.1. A function $f: \Omega \times R^{m} \times R^{k} \longrightarrow R \cup\{+\infty\}$ is called $L \otimes$ $B$-measurable, if it is measurable with respect to the $\sigma$-algebra generated by products of measurable subsets of $\Omega$ and Borel subsets of $R^{m} \times R^{k}$.

Definition 2.2. A function $f: \Omega \times R^{m} \times R^{k} \longrightarrow R$ is a Carathédory function, if

(i) $f(\cdot, u, P)$ is measurable for every $u \in R^{m}, P \in R^{k}$,

(ii) $f(x, \cdot, \cdot)$ is continuous for almost every $x \in \Omega$.

Definition 2.3. A sequence of functions $f_{M}: \Omega \times R^{m} \times R^{k} \longrightarrow R \cup\{+\infty\}$ is said to converge to $f: \Omega \times R^{m} \times R^{k} \longrightarrow R \cup\{+\infty\}$ locally uniformly in $\Omega \times R^{m} \times R^{k}$, if there exists a sequence of measurable subsets $\Omega_{l} \subset \Omega$ with $\operatorname{meas}_{n}\left(\Omega \backslash \Omega_{l}\right) \rightarrow 0$ 
as $l \rightarrow \infty$ such that, for each $l$ and any compact subset $G \subset R^{m} \times R^{k}$,

$$
f_{M}(x, u, P) \rightarrow f(x, u, P) \text { unifomly on } \Omega_{l} \times G \text { as } M \rightarrow+\infty \text {. }
$$

Throughout the rest of this paper $\rightarrow$ denotes the weak convergence of the sequences. The following theorem is a special case of a more general theorem given by Li in [16].

Theorem 2.1. Let $1 \leqslant p \leqslant+\infty$ and $1 \leqslant q \leqslant+\infty$. Let $f: \Omega \times R^{m} \times R^{k} \longrightarrow R$ satisfy

(i) $f(\cdot, \cdot, \cdot)$ is a Carathédory function,

(ii) $f(x, u, \cdot)$ is convex,

(iii) $f(x, u, P) \geqslant a(x), a(\cdot) \in L^{1}(\Omega)$.

Let $f_{M}: \Omega \times R^{m} \times R^{k} \longrightarrow R$ satisfy

(a) $f_{M}(\cdot, \cdot, \cdot)$ are $L \otimes B$-measurable,

(b) $f_{M} \rightarrow f$ locally uniformly in $\Omega \times R^{m} \times R^{k}$,

(c) $f_{M}(x, u, P) \geqslant b(x)$, for some $b(\cdot) \in L^{1}(\Omega)$.

Let $\left\{u_{M}\right\}, u \in L^{p}\left(\Omega ; R^{m}\right)$ and $\left\{P_{M}\right\}, P \in L^{q}\left(\Omega ; R^{k}\right)$ be such that

$$
u_{M} \rightarrow u \text { in } L^{p}\left(\Omega ; R^{m}\right) \text { and } P_{M} \rightarrow P \text { in } L^{q}\left(\Omega ; R^{k}\right) .
$$

Then

$$
\int_{\Omega} f(x, u, P) d x \leqslant \liminf _{M \rightarrow+\infty} \int_{\Omega} f_{M}\left(x, u_{M}, P_{M}\right) d x
$$

\section{The truncation method And the CONVERGEnCE THEOREMS}

Assume that the integrand $f$ satisfies the following hypotheses.

(H1) $f: \Omega \times R^{m} \times R^{m n} \rightarrow R$ is a Carathédory function;

(H2) There exists a $a(\cdot) \in L^{1}(\Omega)$ such that $f(x, u, P) \geqslant a(x)$ for all $(x, u, P) \in$ $\Omega \times R^{m} \times R^{m n}$

(H3) $f(x, u, \cdot)$ is convex for all $(x, u) \in \Omega \times R^{m}$;

(H4) Let $g_{K}(x)=\sup _{|u| \leqslant K,|P| \leqslant K}|f(x, u, P)|$, then $g_{K}(\cdot) \in L^{1}(\Omega)$.

By (H2), without loss of generality, we may assume that $f$ is non-negative. Let $\mathcal{T}^{h}$ be a regular triangulation [6] of $\Omega$ with $h$ being the mesh size and let $\bar{\Omega}_{h}=$ $\bigcup_{K \in \mathcal{T}^{h}} K$. For simplicity, assume $\Omega_{h}=\Omega$ and $\partial \Omega_{0 h}=\partial \Omega_{0}$, where $\partial \Omega_{0 h}$ is the 
union of all $(n-1)$-dimensional faces in $\mathcal{T}^{h}$ whose interior have a nonempty intersection with $\partial \Omega_{0}$. Define

$$
\begin{aligned}
& \mathcal{A}_{h}=\left\{u \in C(\bar{\Omega}):\left.u\right|_{K} \text { is affine, } \forall K \in \mathcal{T}^{h}\right\}, \\
& \mathcal{A}_{h}\left(u_{0 h} ; \partial \Omega_{0}\right)=\left\{u \in \mathcal{A}_{h}: u=u_{0 h} \text { on } \partial \Omega_{0}\right\},
\end{aligned}
$$

where $u_{0 h} \in \mathcal{A}_{h}$ satisfy

$$
u_{0 h} \rightarrow u_{0} \quad \text { in } W^{1, p}\left(\Omega ; R^{m}\right), \quad \text { as } h \rightarrow 0 .
$$

The truncation method for computing the minimizer of $I(\cdot)$ in $\mathcal{A}^{p}$ is to solve the finite problem of minimizing

$$
I_{M}\left(u_{h}\right)=\int_{\Omega} f_{M}\left(x, u_{h}, D u_{h}\right) d x
$$

in $\mathcal{A}_{h}\left(u_{0 h} ; \partial \Omega_{0}\right)$, where $f_{M}$ is a truncation function of $f$ which replaces $f$ by certain slower growth functions on regions where the function $u_{h}$, especially its gradient $D u_{h}$ is so large that the growth of the integrand may be out of control. The theory established in this section on the truncation method provides us with a guide on how to determine the truncation regions and the slower growth substitution functions so that the computation can be successful and efficient.

Let $\left\{\mathcal{T}^{h_{M}}\right\}_{M=1}^{\infty}$ be a given family of regular triangulations of $\Omega$ with $h_{M} \rightarrow 0$ as $M \rightarrow+\infty$. Denote

$$
T_{n}^{h_{M}}=\left\{K: K \text { is a } n \text {-dimensional element in } \mathcal{T}^{h_{M}}\right\} .
$$

Lemma 3.1. Let $1 \leqslant p<+\infty$. Let $\widetilde{T}_{n}^{h_{M}}$ be subsets of $T_{n}^{h_{M}}$ such that the sets $\widetilde{\Omega}_{h_{M}}=\bigcup_{K \in \widetilde{T}_{n}^{h_{M}}} \bar{K}$ satisfy

$$
\sum_{M=1}^{+\infty} \operatorname{meas}_{n}\left(\widetilde{\Omega}_{h_{M}}\right)<+\infty
$$

Let the truncation function $f_{M}(x, u, P ; p)$ be defined by

$$
f_{M}(x, u, P ; p)= \begin{cases}f(x, u, P), & x \in \Omega \backslash \widetilde{\Omega}_{h_{M}} \\ \min \left\{\alpha_{h_{M}}(x)\left(1+|P|^{p}\right), f(x, u, P)\right\}, & x \in \widetilde{\Omega}_{h_{M}},\end{cases}
$$

where $\alpha_{h_{M}} \in L^{\infty}(\Omega)$ and $\bar{\alpha}_{h_{M}} \geqslant \alpha_{h_{M}}(x) \geqslant \alpha_{1}>0$, a.e. in $\Omega$. Then

(a) $f_{M}(\cdot, \cdot, \cdot ; p)$ are $L \otimes B$-measurable; 
(b) $f_{M}(x, u, P ; p) \geqslant b(x)$, for some $b(\cdot) \in L^{1}(\Omega)$;

(c) $f_{M} \rightarrow f$ locally uniformly in $\Omega \times R^{m} \times R^{m n}$.

Proof. Since both $f(x, u, P)$ and $\alpha_{h_{M}}(x)\left(1+|P|^{p}\right)$ are Carathédory functions and hence $L \otimes B$-measurable, it is not difficult to verify that $f_{M}(\cdot, \cdot, \cdot ; p)$ are $L \otimes B$ measurable.

Take $b(x)=\min \left\{a(x), \alpha_{1}\right\}$, then $b(\cdot) \in L^{1}(\Omega)$ and $f_{M}(x, u, P ; p) \geq b(x)$.

To prove (c), let

$$
\Omega_{l}=\Omega \backslash\left(\bigcup_{j=l}^{+\infty} \widetilde{\Omega}_{h_{j}}\right)
$$

It is obvious that $\Omega_{l}$ are measurable and $\Omega_{l} \subset \Omega$. By (3.2) and (3.4), we have

$$
\operatorname{meas}_{n}\left(\Omega \backslash \Omega_{l}\right) \leqslant \sum_{j=l}^{+\infty} \operatorname{meas}_{n}\left(\widetilde{\Omega}_{h_{j}}\right) \rightarrow 0, \text { as } l \rightarrow+\infty .
$$

It follows from (3.3) and (3.4) that, for each $l$,

$$
f_{M}(x, u, P ; p)=f(x, u, P), \forall x \in \Omega_{l} \text { as long as } M>l .
$$

This completes the proof.

Corollary 3.1. Let $1 \leqslant p<+\infty$. Let $f_{M}$ be given by (3.3) with $\widetilde{\Omega}_{h_{M}}$ satisfying (3.2). Define

$$
I_{M}(u ; p)=\int_{\Omega} f_{M}(x, u, D u ; p) d x .
$$

Let $\left\{u_{M}\right\}, u \in W^{1, p}\left(\Omega ; R^{m}\right)$ be such that

$$
u_{M} \rightarrow u \text { in } W^{1, p}\left(\Omega ; R^{m}\right), \quad \text { as } M \rightarrow+\infty .
$$

Then

$$
I(u) \leq \liminf _{M \rightarrow+\infty} I_{M}\left(u_{M} ; p\right)
$$

Proof. The conclusion follows directly from Theorem 2.1 and Lemma 3.1.

Definition 3.1. $u \in W^{1, p}\left(\Omega ; R^{m}\right)(1 \leqslant p<+\infty)$ is said to be a partial regular function with singular set $E(u)$, if $D u \in L^{\infty}\left(\Omega \backslash F ; R^{m n}\right)$ for any open set $F \supset$ $E(u)$, and $D u \notin L^{\infty}\left(\Omega \backslash G ; R^{m n}\right)$ for any open set $G$ such that $G \cap E(u) \neq \emptyset$. 
In what follows in this paper, to simplify the notation, we denote by $E$ a set with zero $n$-dimensional Lebesgue measure and finite $(n-1)$-dimensional Hausdorff measure, especially we always assume that the singular set $E(u)$ in question satisfies meas $_{n} E(u)=0$ and its $(n-1)$-dimensional Hausdorff measure is finite.

Definition 3.2. Let $E$ be given. The sequence of sets $\widetilde{\Omega}_{h_{M}}^{E}=\bigcup_{K \in \widetilde{T}_{n}^{h_{M}(E)}} \bar{K}$, where $\widetilde{T}_{n}^{h_{M}}(E) \subset T_{n}^{h_{M}}$, is called an admissible finite element covering of $E$ if there exist $0<C_{2}\left(h_{M}\right) \leqslant C_{1}\left(h_{M}\right)$ satisfying $\sum_{M=1}^{+\infty} C_{1}\left(h_{M}\right)<+\infty$ such that

(D1) $E \subset \widetilde{\Omega}_{h_{M}}^{E}$,

(D2) $\forall K \in \widetilde{\Omega}_{h_{M}}^{E}, \operatorname{dist}(E, K) \leqslant C_{1}\left(h_{M}\right)$,

(D3) $\forall K \in \Omega \backslash \widetilde{\Omega}_{h_{M}}^{E}, \operatorname{dist}(E, K) \geqslant C_{2}\left(h_{M}\right)$,

where $\operatorname{dist}(E, K)$ is the distance between the two sets.

Definition 3.3. Let $1 \leqslant p<+\infty$. The sequence of truncation functionals

$$
I_{M}^{E}(u ; p)=\int_{\Omega} f_{M}^{E}(x, u, D u ; p) d x,
$$

where the truncation functions $f_{M}^{E}$ are defined by

$$
f_{M}^{E}(x, u, P ; p)= \begin{cases}f(x, u, P), & x \in \Omega \backslash \widetilde{\Omega}_{h_{M}}^{E} \\ \min \left\{\alpha_{h_{M}}(x)\left(1+|P|^{p}\right), f(x, u, P)\right\}, & x \in \widetilde{\Omega}_{h_{M}}^{E},\end{cases}
$$

with $\alpha_{h_{M}} \in L^{\infty}(\Omega)$ and $\bar{\alpha}_{h_{M}} \geqslant \alpha_{h_{M}}(x) \geqslant \alpha_{1}>0$ a.e. in $\Omega$, is said to be consistent with the set $E$ if $\left\{\widetilde{\Omega}_{h_{M}}^{E}\right\}$ is an admissible finite element covering of $E$.

Lemma 3.2. Let $1 \leqslant p<+\infty$. Let $\widetilde{u} \in W^{1, p}\left(\Omega ; R^{m}\right)$ be a partially regular function with singular set $E(\widetilde{u}) \in E$ and satisfy $f(x, \widetilde{u}, D \widetilde{u}) \in L^{1}(\Omega)$. Let $I_{M}^{E}(\cdot ; p)$ be consistent with the singular set $E$ with $\left\{\widetilde{\Omega}_{h_{M}}^{E}\right\}$ being the corresponding admissible finite element covering of $E$. Let $u_{h} \in \mathcal{A}_{h}\left(u_{0 h} ; \partial \Omega_{0}\right)$ satisfy

$$
u_{h} \rightarrow \widetilde{u} \text { in } W^{1, p}\left(\Omega ; R^{m}\right), \quad \text { as } h \rightarrow 0,
$$

and be uniformly bounded in $W^{1, \infty}\left(\Omega \backslash \widetilde{\Omega}_{h_{M}}^{E} ; R^{m}\right)$ for each $M$. Then for any $\varepsilon>0$, there exist a non-increasing function $M(\varepsilon)>0$ and a function $h(\varepsilon, M)>0$ with 
$h(\cdot, M)$ non-decreasing and $h(\varepsilon, \cdot)$ non-increasing such that

$$
\left|I_{M}^{E}\left(u_{h} ; p\right)-I(\widetilde{u})\right|<\varepsilon, \quad \text { if } M>M(\varepsilon) \text { and } 0<h<h(\varepsilon, M) .
$$

Proof.

$$
\begin{aligned}
I_{M}^{E}\left(u_{h} ; p\right)-I(\widetilde{u})= & \int_{\Omega}\left[f_{M}^{E}(x, \widetilde{u}, D \widetilde{u} ; p)-f(x, \widetilde{u}, D \widetilde{u})\right] d x \\
& +\int_{\Omega}\left[f_{M}^{E}\left(x, u_{h}, D u_{h} ; p\right)-f_{M}^{E}(x, \widetilde{u}, D \widetilde{u} ; p)\right] d x \\
\triangleq & I_{1}(M)+I_{2}(h, M) .
\end{aligned}
$$

By (3.6), we have

$$
\begin{aligned}
\left|I_{1}(M)\right| & =\left|\int_{\widetilde{\Omega}_{h_{M}}^{E}}\left[f_{M}^{E}(x, \widetilde{u}, D \widetilde{u} ; p)-f(x, \widetilde{u}, D \widetilde{u})\right] d x\right| \\
& \leqslant 2 \int_{\widetilde{\Omega}_{h_{M}}^{E}}|f(x, \widetilde{u}, D \widetilde{u})| d x .
\end{aligned}
$$

It follows from $f(x, \widetilde{u}, D \widetilde{u}) \in L^{1}(\Omega)$ that for any $\varepsilon>0$, there exists a $\delta_{1}(\varepsilon)>0$, such that

$$
\int_{\Omega^{\prime}}|f(x, \widetilde{u}, D \widetilde{u})| d x<\varepsilon, \quad \forall \Omega^{\prime} \subset \Omega \text { with } \operatorname{meas}_{n}\left(\Omega^{\prime}\right)<\delta_{1}(\varepsilon) .
$$

Since the $(n-1)$-dimensional Hausdorff measure of $E$ is finite and $\left\{\widetilde{\Omega}_{h_{M}}^{E}\right\}$ is an admissible finite element covering of $E$, we have $\lim _{M \rightarrow+\infty} \operatorname{meas}_{n}\left(\widetilde{\Omega}_{h_{M}}^{E}\right)=0$. Thus, by (3.9) and (3.10), there exists a non-increasing positive function $M(\cdot)$ such that

$$
\operatorname{meas}_{n}\left(\widetilde{\Omega}_{h_{M}}^{E}\right)<\delta_{1}\left(\frac{\varepsilon}{4}\right) \text { and }\left|I_{1}(M)\right|<\frac{\varepsilon}{2}, \quad \forall M>M(\varepsilon) .
$$

By (3.6), we have

$$
\begin{aligned}
I_{2}(h, M)= & \int_{\widetilde{\Omega}_{h_{M}}^{E}}\left[f_{M}^{E}\left(x, u_{h}, D u_{h} ; p\right)-f_{M}^{E}(x, \widetilde{u}, D \widetilde{u} ; p)\right] d x \\
& +\int_{\Omega \backslash \widetilde{\Omega}_{h_{M}}^{E}}\left[f\left(x, u_{h}, D u_{h}\right)-f(x, \widetilde{u}, D \widetilde{u})\right] d x \\
\triangleq & I_{21}(h, M)+I_{22}(h, M) .
\end{aligned}
$$


To estimate $I_{21}(h, M)$, we first notice that, as a consequence of $(3.7),\left|D u_{h}\right|^{p}$ are equi-integrable on $\Omega$, and thus, for any $\varepsilon>0$ and given $\bar{\alpha}_{h_{M}} \geqslant \alpha_{1}>0$, there exists a $\delta_{2}(\varepsilon, M)>0$, such that for any $\Omega^{\prime} \subset \Omega$, we have

$$
\int_{\Omega^{\prime}} \bar{\alpha}_{h_{M}}\left|D u_{h}\right|^{p} d x<\varepsilon, \quad \forall h>0 \quad \text { if } \operatorname{meas}_{n}\left(\Omega^{\prime}\right)<\delta_{2}(\varepsilon, M) .
$$

We claim that for any $\varepsilon>0, M>0$, there exists a $h_{1}(\varepsilon, M)>0$ with $h_{1}(\cdot, M)$ non-decreasing and $h_{1}(\varepsilon, \cdot)$ non-increasing such that

$$
\left|I_{21}(h, M)\right|<\frac{\varepsilon}{4}, \quad \forall h \in\left(0, h_{1}(\varepsilon, M)\right) .
$$

Suppose otherwise. Then, there would be $\varepsilon_{0}>0, M_{0}>0$ and a decreasing sequence $\left\{h_{j}^{0}\right\}$ with $\lim _{j \rightarrow+\infty} h_{j}^{0}=0$ such that $\left|I_{21}\left(h_{j}^{0}, M_{0}\right)\right| \geqslant \frac{\varepsilon_{0}}{4}$ for all $j$. By (3.7), without loss of generality, we may assume

$$
u_{h_{j}^{0}} \rightarrow \widetilde{u} \text { and } D u_{h_{j}^{0}} \rightarrow D \widetilde{u} \text { a.e. in } \Omega
$$

and thus, by (3.6) and (H1), we have

$$
\left[f_{M_{0}}^{E}\left(x, u_{h_{j}^{0}}, D u_{h_{j}^{0}} ; p\right)-f_{M_{0}}^{E}(x, \widetilde{u}, D \widetilde{u} ; p)\right] \rightarrow 0 \quad \text { a.e. } x \in \Omega
$$

Let

$G\left(\varepsilon_{0}, M_{0}, h_{j}^{0}\right)=\left\{x \in \Omega:\left|f_{M_{0}}^{E}\left(x, u_{h_{j}^{0}}, D u_{h_{j}^{0}} ; p\right)-f_{M_{0}}^{E}(x, \widetilde{u}, D \widetilde{u} ; p)\right| \geqslant \frac{\varepsilon_{0}}{16 \operatorname{meas}_{n}(\Omega)}\right\}$

By (3.14), there exists $J_{0}=J\left(\varepsilon_{0}, M_{0}\right)>0$ such that

$$
\operatorname{meas}_{n}\left(G\left(\varepsilon_{0}, M_{0}, h_{j}^{0}\right)\right)<\min \left\{\frac{\varepsilon_{0}}{16 \bar{\alpha}_{h_{M_{0}}}}, \delta_{1}\left(\frac{\varepsilon_{0}}{16}\right), \delta_{2}\left(\frac{\varepsilon_{0}}{16}, M_{0}\right)\right\}, \quad \forall j>J_{0}
$$


As a consequence of (3.6), (3.10), (3.12) and (3.15), we have

$$
\begin{aligned}
\left|I_{21}\left(h_{j}^{0}, M_{0}\right)\right| & \leqslant \int_{\widetilde{\Omega}_{h_{M_{0}}}^{E} \cap G\left(\varepsilon_{0}, M_{0}, h_{j}^{0}\right)}\left(\bar{\alpha}_{h_{M_{0}}}\left(1+\left|D u_{h_{j}^{0}}\right|^{p}\right)+|f(x, \widetilde{u}, D \widetilde{u})|\right) d x \\
& +\int_{\widetilde{\Omega}_{h_{M_{0}}}^{E} \cap\left(\Omega \backslash G\left(\varepsilon_{0}, M_{0}, h_{j}^{0}\right)\right)}\left|f_{M_{0}}^{E}\left(x, u_{h_{j}^{0}}, D u_{h_{j}^{0}} ; p\right)-f_{M_{0}}^{E}(x, \widetilde{u}, D \widetilde{u} ; p)\right| d x \\
& \leqslant \frac{\varepsilon_{0}}{16 \bar{\alpha}_{h_{M_{0}}}} \bar{\alpha}_{h_{M_{0}}}+\frac{\varepsilon_{0}}{8}+\frac{\varepsilon_{0}}{16 \operatorname{meas}_{n}(\Omega)} \operatorname{meas}_{n}\left(\Omega \backslash G\left(\varepsilon_{0}, M_{0}, h_{j}^{0}\right)\right) \\
& <\frac{\varepsilon_{0}}{4}, \quad \forall j>J_{0} .
\end{aligned}
$$

This is a contradiction.

We also claim that for any $\varepsilon>0, M>0$, there exists a $h_{2}(\varepsilon, M)>0$ with $h_{2}(\cdot, M)$ non-decreasing and $h_{2}(\varepsilon, \cdot)$ non-increasing such that

$$
\left|I_{22}(h, M)\right|<\frac{\varepsilon}{4}, \quad \forall h \in\left(0, h_{2}(\varepsilon, M)\right) .
$$

Suppose otherwise. Then, there would be $\varepsilon_{1}>0, M_{1}>0$ and a decreasing sequence $\left\{h_{j}^{1}\right\}$ with $\lim _{j \rightarrow+\infty} h_{j}^{1}=0$ such that $\left|I_{22}\left(h_{j}^{1}, M_{1}\right)\right| \geqslant \frac{\varepsilon_{1}}{4}$ for all $j$. By (3.7), without loss of generality, we may assume

$$
u_{h_{j}^{1}} \rightarrow \widetilde{u} \text { and } D u_{h_{j}^{1}} \rightarrow D \widetilde{u} \text { a.e. in } \Omega
$$

and thus, by (H1), we have

$$
\left[f\left(x, u_{h_{j}^{1}}, D u_{h_{j}^{1}}\right)-f(x, \widetilde{u}, D \widetilde{u})\right] \rightarrow 0 \text { a.e. } x \in \Omega .
$$

By (H4) and noticing that by assumption there exists a $C\left(M_{1}\right)>0$ such that

$$
\left|u_{h}(x)\right| \leqslant C\left(M_{1}\right) \text { and }\left|D u_{h}(x)\right| \leqslant C\left(M_{1}\right) \text { a.e. } x \in \Omega \backslash \widetilde{\Omega}_{h_{M_{1}}}^{E}, \forall h,
$$

we have

$$
\left|f\left(x, u_{h_{j}^{1}}, D u_{h_{j}^{1}}\right)-f(x, \widetilde{u}, D \widetilde{u})\right| \leqslant g_{C\left(M_{1}\right)}(x)+|f(x, \widetilde{u}, D \widetilde{u})| \in L^{1}\left(\Omega \backslash \widetilde{\Omega}_{h_{M_{1}}}^{E}\right) .
$$

It follows from (3.18), (3.19) and the dominated convergence theorem [11] that

$$
\lim _{j \rightarrow+\infty}\left|I_{22}\left(h_{j}^{1}, M_{1}\right)\right|=0 .
$$

This is a contradiction. 
Now, (3.8) follows as a consequence of (3.11), (3.13) and (3.17) with $h(\varepsilon, M)=$ $\min \left\{h_{1}(\varepsilon, M), h_{2}(\varepsilon, M), h_{M}\right\}$. This completes the proof.

Theorem 3.1. Let $1 \leqslant p<+\infty$. Let

$$
\mathcal{A}_{E}^{p}=\left\{u \in \mathcal{A}^{p}: u \text { is partial regular with singular set } E(u) \subset E\right\} .
$$

Let $I_{M}^{E}(\cdot ; p)$ be consistent with the set $E$, with $\left\{\widetilde{\Omega}_{h_{M}}^{E}\right\}$ being the corresponding admissible finite element covering of $E$. Then for any $\varepsilon>0$, there exist a nonincreasing function $M(\varepsilon)>0$ and a function $h(\varepsilon, M)>0$ with $h(\cdot, M)$ nondecreasing and $h(\varepsilon, \cdot)$ non-increasing such that

$$
\inf _{u_{h} \in \mathcal{A}_{h}\left(u_{0 h} ; \partial \Omega_{0}\right)} I_{M}^{E}\left(u_{h} ; p\right)<\inf _{u \in \mathcal{A}_{E}^{p}} I(u)+2 \varepsilon, \quad \text { if } M>M(\varepsilon) \text { and } 0<h<h(\varepsilon, M) .
$$

Proof. Without loss of generality, we assume that, for any $\varepsilon>0$, there exists a $\widetilde{u}_{\varepsilon} \in \mathcal{A}_{E}^{p}$ such that

$$
I\left(\widetilde{u}_{\varepsilon}\right)<\inf _{u \in \mathcal{A}_{E}^{p}} I(u)+\varepsilon<+\infty .
$$

Extending $\widetilde{u}_{\varepsilon}$ to $W_{0}^{1, p}\left(R^{n} ; R^{m}\right)$ by the extension theorem for Sobolev spaces [1], recalling that $\widetilde{u}_{\varepsilon} \in W^{1, \infty}\left(\Omega \backslash F ; R^{m}\right)$ for any open set $E \subset F \subset \Omega$, we may assume that $\widetilde{u}_{\varepsilon} \in W^{1, \infty}\left(R^{n} \backslash F ; R^{m}\right)$ for any open set $E \subset F \subset R^{n}$. Thus, by the denseness of smooth functions in $W_{0}^{1, p}\left(R^{n} ; R^{m}\right)$ [1] and the standard finite approximation theories [6], there exist $u_{h}^{\varepsilon} \in \mathcal{A}_{h}\left(u_{0 h} ; \partial \Omega_{0}\right)$ such that $u_{h}^{\varepsilon}$ are uniformly bounded in $W^{1, \infty}\left(\Omega \backslash \widetilde{\Omega}_{h_{M}}^{E} ; R^{m}\right)$ for each $M$ and

$$
u_{h}^{\varepsilon} \rightarrow \widetilde{u}_{\varepsilon} \quad \text { in } W^{1, p}\left(\Omega ; R^{m}\right), \quad \text { as } h \rightarrow 0 .
$$

Hence, from Lemma 3.2 and (3.22), the conclusion of the theorem follows.

With the above preparations, we can proceed to present our main results, which briefly speaking conclude that a uniformly bounded sequence of finite element solutions leads to a minimizer (for the case 1 and case 3 ), while an unbounded one provides a minimizing sequence (for the case 2 and case 4 ).

Theorem 3.2. Suppose that $\hat{u} \in W^{1, q}\left(\Omega ; R^{m}\right)(1 \leqslant q<+\infty)$ is a minimizer of $I(\cdot)$ in $\mathcal{A}^{p}(1 \leqslant p \leqslant q)$ and $\hat{u} \in \mathcal{A}_{E}^{p}$ (see (3.20)). Let $\left\{\varepsilon_{j}\right\}$ be a decreasing sequence 
with $\lim _{j \rightarrow \infty} \varepsilon_{j}=0$. Let $I_{M}^{E}(\cdot ; p)$ be consistent with the singular set $E$ with $\left\{\widetilde{\Omega}_{h_{M}}^{E}\right\}$ being the corresponding admissible finite element covering of $E$. Then

(1) There exist a non-increasing function $M(\varepsilon)>0$ and a function $h(\varepsilon, M)>$ 0 with $h(\cdot, M)$ non-decreasing and $h(\varepsilon, \cdot)$ non-increasing such that

$$
\inf _{u \in \mathcal{A}_{h}\left(u_{0 h} ; \partial \Omega_{0}\right)} I_{M}^{E}(u ; p)<I(\hat{u})+\varepsilon_{j}, \forall M \geqslant M\left(\varepsilon_{j}\right) \text { and } \forall h \in\left(0, h\left(\varepsilon_{j}, M\right)\right),
$$

and, for all $M \geqslant M\left(\varepsilon_{j}\right)$ and $0<h \leqslant h\left(\varepsilon_{j}, M\right)$, there exist $u_{h}^{\varepsilon_{j}} \in \mathcal{A}_{h}\left(u_{0 h} ; \partial \Omega_{0}\right)$ such that $u_{h}^{\varepsilon_{j}}$ are uniformly bounded in $W^{1, \infty}\left(\Omega \backslash \widetilde{\Omega}_{h_{M}}^{E} ; R^{m}\right)$ for each $M$ and

$$
I_{M}^{E}\left(u_{h}^{\varepsilon_{j}} ; p\right)<I(\hat{u})+2 \varepsilon_{j}, \forall M \geqslant M\left(\varepsilon_{j}\right) \text { and } \forall h \in\left(0, h\left(\varepsilon_{j}, M\right)\right) .
$$

(2) Let $M_{j} \geqslant M\left(\varepsilon_{j}\right), 0<h^{j} \leqslant h\left(\varepsilon_{j}, M_{j}\right)$. Let $\bar{u}_{j} \in \mathcal{A}_{h^{j}}\left(u_{0 h^{j}} ; \partial \Omega_{0}\right)$ be minimizers of $I_{M_{j}}^{E}(\cdot ; p)$ in $\mathcal{A}_{h^{j}}\left(u_{0 h^{j}} ; \partial \Omega_{0}\right)$. Suppose that the sequence $\left\{\bar{u}_{j}\right\}_{j=1}^{+\infty}$ is sequentially weakly precompact in $W^{1, r}\left(\Omega ; R^{m}\right)$ for some $p \leqslant r \leqslant q$. Then, there exists a function $\bar{u} \in \mathcal{A}^{r}$ and a subsequence of $\left\{\bar{u}_{j}\right\}_{j=1}^{+\infty}$, again denoted by $\left\{\bar{u}_{j}\right\}_{j=1}^{+\infty}$, such that

$$
\bar{u}_{j} \rightarrow \bar{u} \text { in } W^{1, r}\left(\Omega ; R^{m}\right)
$$

and

$$
I(\bar{u})=\inf _{u \in \mathcal{A}^{p}} I(u)=\lim _{j \rightarrow+\infty} I_{M_{j}}^{E}\left(\bar{u}_{j} ; p\right) .
$$

Proof. The conclusion (1) of the theorem follows from a similar argument as in the proof of Theorem 3.1.

(3.25) is a consequence of the sequentially weakly precompactness of the sequence $\left\{\bar{u}_{j}\right\}_{j=1}^{+\infty}$, and it follows from

$$
\left.\bar{u}_{j}\right|_{\partial \Omega_{0}}=u_{0 h^{j}} \text { and } u_{0 h^{j}} \rightarrow u_{0} \text { in } L^{r}\left(\partial \Omega ; R^{m}\right),
$$

that $\left.\bar{u}\right|_{\partial \Omega_{0}}=u_{0}$ and thus $\bar{u} \in \mathcal{A}^{r} \subset \mathcal{A}^{p}$. Hence, by Corollary 3.1 we have

$$
I(\bar{u}) \leqslant \liminf _{j \rightarrow+\infty} I_{M_{j}}^{E}\left(\bar{u}_{j} ; p\right) .
$$

On the other hand, by (3.23), we have

$$
I_{M_{j}}^{E}\left(\bar{u}_{j} ; p\right)=\inf _{u \in \mathcal{A}_{h^{j}}\left(u_{0 h^{j}} ; \partial \Omega_{0}\right)} I_{M_{j}}^{E}(u ; p)<I(\hat{u})+\varepsilon_{j},
$$


and thus

$$
\limsup _{j \rightarrow+\infty} I_{M_{j}}^{E}\left(\bar{u}_{j} ; p\right) \leqslant I(\hat{u})=\inf _{u \in \mathcal{A}^{p}} I(u) .
$$

This and (3.27) lead to (3.26).

Remark 3.1. When $r>1$, the boundedness of $\left\{\bar{u}_{j}\right\}_{j=1}^{+\infty}$ in $W^{1, r}\left(\Omega ; R^{m}\right)$ implies that the sequence is sequentially weakly precompact in $W^{1, r}\left(\Omega ; R^{m}\right)$. While in the case when $r=p=1$, if the singular set $E$ is a $(n-k)$-dimensional locally Lipschitz continuous manifolds for some $k \geqslant 1$ with meas $_{n-k}(E)<\infty$ and there exists a non-increasing continuous function $\xi:(0,+\infty) \rightarrow(0,+\infty)$ satisfying $\int_{0}^{1} \xi(\rho) d \rho<\infty$ such that

$$
\left|D \bar{u}_{j}(x)\right| \leqslant \rho^{1-k} \xi(\rho), \text { if } \operatorname{dist}(x, E) \geqslant \rho>0, \forall j,
$$

then the sequence $\left\{\bar{u}_{j}\right\}_{j=1}^{+\infty}$ is sequentially weakly precompact in $W^{1,1}\left(\Omega ; R^{m}\right)$.

Theorem 3.3. Let $1<p<+\infty$. Let $\left\{L_{i}\right\}_{i=1}^{+\infty}$ be an increasing sequence satisfying $\lim _{i \rightarrow+\infty} L_{i}=+\infty$. Define

$$
\begin{aligned}
& \mathcal{A}^{p}\left(L_{i}\right)=\left\{u \in \mathcal{A}^{p}:|u|_{1, p}^{p} \leqslant L_{i}\right\}, \\
& \mathcal{A}_{h}^{p}\left(L_{i}\right)=\left\{u \in \mathcal{A}_{h}\left(u_{0 h} ; \partial \Omega_{0}\right):|u|_{1, p}^{p} \leqslant L_{i}\right\} .
\end{aligned}
$$

For each $i>0$, suppose that $\hat{u}^{i} \in W^{1, p}\left(\Omega ; R^{m}\right)$ is a minimizer of $I(\cdot)$ in $\mathcal{A}^{p}\left(L_{i}\right)$ and $\hat{u}^{i} \in \mathcal{A}_{E}^{p}$ (see (3.20)). Let $\left\{\varepsilon_{j}\right\}$ be a decreasing sequence with $\lim _{j \rightarrow \infty} \varepsilon_{j}=0$. Then, for each $i>0$,

(1) There exist a non-increasing function $M(\varepsilon)>0$ and a function $h(\varepsilon, M)>$ 0 with $h(\cdot, M)$ non-decreasing and $h(\varepsilon, \cdot)$ non-increasing such that

$$
\inf _{u \in \mathcal{A}_{h}^{p}\left(L_{i}\right)} I_{M}^{E}(u ; p)<I\left(\hat{u}^{i}\right)+\varepsilon_{j}, \quad \text { if } M \geqslant M\left(\varepsilon_{j}\right) \text { and } 0<h \leqslant h\left(\varepsilon_{j}, M\right) .
$$

(2) Let $M_{j} \geqslant M\left(\varepsilon_{j}\right), 0<h^{j} \leqslant h\left(\varepsilon_{j}, M_{j}\right)$. Let $\bar{u}_{j}^{i} \in \mathcal{A}_{h^{j}}^{p}\left(L_{i}\right)$ be minimizers of $I_{M_{j}}^{E}(\cdot ; p)$ in $\mathcal{A}_{h^{j}}^{p}\left(L_{i}\right)$. Then there exist a function $\bar{u}^{i} \in \mathcal{A}^{p}\left(L_{i}\right)$ and a subsequence of $\left\{\bar{u}_{j}^{i}\right\}_{j=1}^{+\infty}$, again denoted by $\left\{\bar{u}_{j}^{i}\right\}_{j=1}^{+\infty}$, such that

$$
\bar{u}_{j}^{i} \rightarrow \bar{u}^{i} \quad \text { in } W^{1, p}\left(\Omega ; R^{m}\right), \quad \text { as } j \rightarrow+\infty,
$$

and

$$
I\left(\bar{u}^{i}\right)=\inf _{u \in \mathcal{A}^{p}\left(L_{i}\right)} I(u)=\lim _{j \rightarrow+\infty} I_{M_{j}}^{E}\left(\bar{u}_{j}^{i} ; p\right) .
$$


(3) There exists a non-decreasing function $j(i)$ satisfying $\lim _{i \rightarrow+\infty} j(i)=+\infty$ such that

$$
\inf _{u \in \mathcal{A}^{p}} I(u)=\lim _{i \rightarrow+\infty} I\left(\bar{u}^{i}\right)=\lim _{i \rightarrow+\infty} I_{M_{j(i)}}^{E}\left(\bar{u}_{j(i)}^{i} ; p\right) .
$$

Proof. For each $i>0$, the conclusion (1) and (2) of the theorem follow from a similar argument as in the proof of Theorem 3.2. The conclusion (3) of the theorem follows from (3.30) and (3.33).

Remark 3.2. The Theorem 3.3 can also be extended to cover the case when $p=1$, as long as there are given sequences of sets $B_{h_{j}, i} \subset \mathcal{A}_{h_{j}}\left(u_{0 h_{j}} ; \partial \Omega_{0}\right)$ such that $\cup_{j=1}^{\infty} B_{h_{j}, i}$ is precompact in $\mathcal{A}^{1}$ for all $i$ and $\overline{\cup_{i, j=1}^{\infty} B_{h_{j}, i}}=\mathcal{A}^{1}$.

Remark 3.3. The condition that the parameter $\alpha_{h_{M}}(x) \geqslant \alpha_{1}>0$ a.e. in $\Omega$ is not essential for the convergence theorems. However, in computation, it helps to guarantee that the numerical solutions are uniformly bounded in $\mathcal{A}^{p}$. Notice also that, unlike that in [14], we do not require that $\alpha_{h_{M}}$ go to infinity as $M \rightarrow \infty$.

Remark 3.4. The singular set $E$ for an absolute minimizer is usually not known in advance when the Lavrentiev phenomenon is involved, and thus it needs to be decided in the process of computation. Fortunately, $E$ is usually contained in the set where the standard finite element solutions have large derivatives.

\section{NumERICAL RESULtS}

4.1. The Manià's example. Consider the problem of minimizing

$$
I(u) \triangleq \int_{0}^{1} f\left(x, u, u^{\prime}\right) d x=\int_{0}^{1}\left(u^{3}-x\right)^{2}\left(u^{\prime}\right)^{6} d x
$$

in $\mathcal{A}^{1}=\left\{u \in W^{1,1}(0,1): u(0)=0, u(1)=1\right\}$, where $\Omega=(0,1)$.

Divide $\Omega$ into $N$ equal parts, then $h=N^{-1}$, denote

$$
x_{i}=i h, \quad i=0,1, \ldots, N ; K_{i}=\left[x_{i-1}, x_{i}\right], \quad i=1,2, \ldots, N,
$$

and

$$
\mathcal{A}_{h}=\left\{u \in C([0,1]):\left.u\right|_{K_{i}} \text { is affine, } \forall i=1,2, \ldots, N ; u(0)=0, u(1)=1\right\} .
$$

Take $1 \leqslant p<1.5$, let $I_{M}^{E}(u ; p)$ be given by (3.5) and (3.6) with $\alpha_{h_{M}}(x) \equiv$ $\bar{\alpha}_{h_{M}}>\alpha_{1}=10^{-4}$. Since the numerical solutions obtained by the standard finite element methods have large derivatives near 0 and 1 , we take $E=\{0,1\}$ and 
$\widetilde{\Omega}_{h_{M}}^{E}=\left\{K_{1}, K_{h_{M}^{-1}}\right\}$ initially. Numerical experiments with various values of the truncation parameter $\bar{\alpha}_{h_{M}}$ show that $\{1\}$ is not a singular point, while $\{0\}$ is indeed a singular point with the Lavrentiev phenomenon. It is interesting to point out here that the numerical experiments show that the mesh does not need to be further refined for a given $\widetilde{\Omega}_{h_{M}}^{E}$. Thus by Theorem 3.2, to guarantee the convergence, $\bar{\alpha}_{h_{M}}$ should be chosen so that $\left\|u_{h_{M}}\right\|_{1, p, K_{1}}$ are uniformly bounded if $1<p<1.5$, and $\left\|u_{h_{M}}\right\|_{1,1, K_{1}} \rightarrow 0$ if $p=1$ as is required by the equi-integrability condition for a precompact set in $W^{1,1}(0,1)$. In our numerical experiments, we use $p=1$ and assume that the leading term of the singularity has the form $\gamma x^{s}$ and evaluate $\gamma>0$ and $s \in(0,1)$ by the least square method using the nodal values of the numerical solutions $\left\{u_{h_{M}}\left(x_{i}\right)\right\}_{i=1}^{3}$ near the singularity. We choose $\bar{\alpha}_{h_{M}}$ so that the $l^{2}$-error between $u_{h_{M}}$ and $\gamma x^{s}$ on $\left\{x_{i}\right\}_{i=1}^{3}$ is minimized.

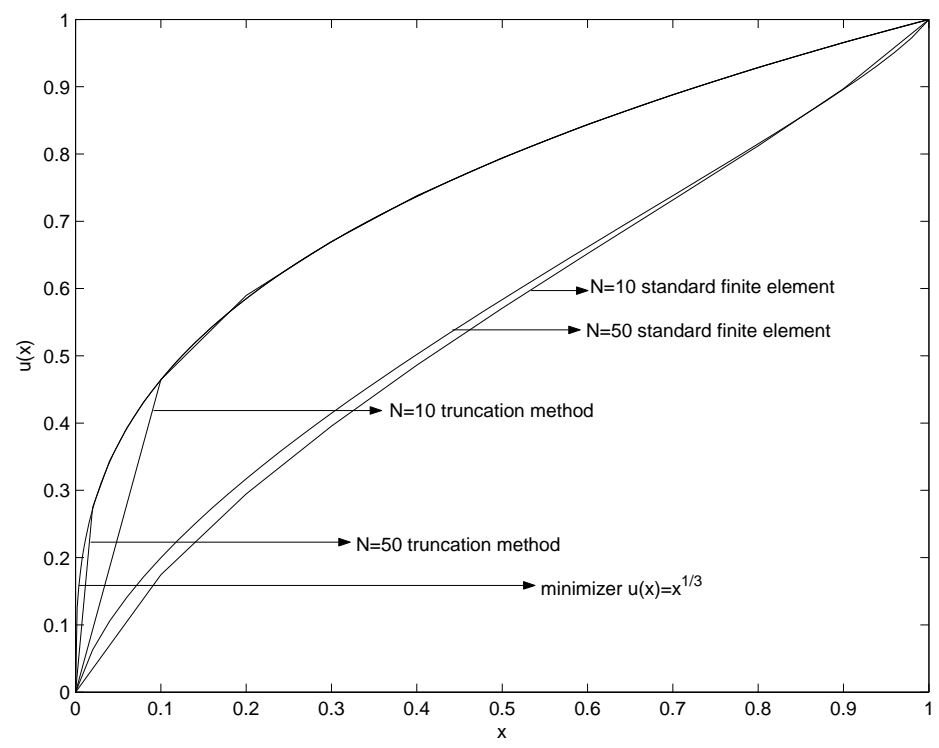

FiguRE 1. Numerical solutions for $h_{1}=0.1$ and $h_{2}=0.02$.

The numerical solutions for $h_{1}=0.1\left(N_{1}=10\right)$ and $h_{2}=0.02\left(N_{2}=50\right)$ obtained by the standard finite element method and the truncation method with $\bar{\alpha}_{h_{1}}=0.0008$ and $\bar{\alpha}_{h_{2}}=0.0009$ respectively are shown in Figure 1 . The convergence behavior of the truncation method is shown in Figure 2. 


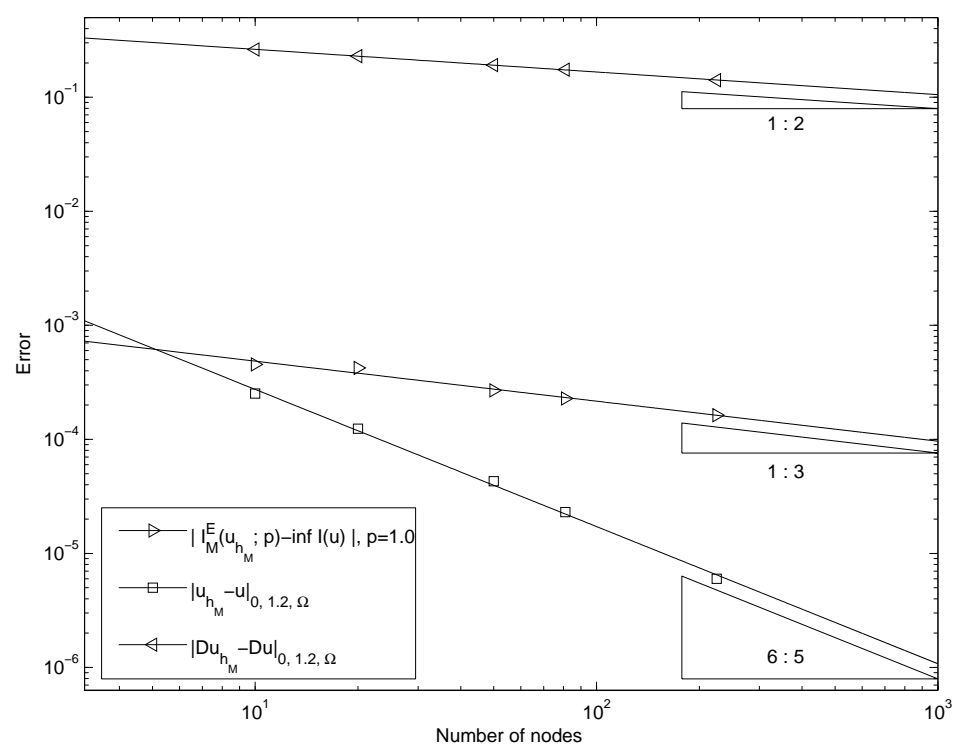

FiguRE 2. Convergence rates of the truncation method for Manià's example.

4.2. A 2-D example with continuous Sobolev exponent dependence. Consider the problem [10] of minimizing the functional

$$
\begin{aligned}
I(u) & \triangleq \int_{\Omega} f(x, y, u, D u) d x d y \\
& =\int_{\Omega} 66\left(\frac{13}{14}\right)^{14}\left(\frac{y}{y-1}\right)^{14}|u|^{\frac{14-3 y}{y-1}}\left(|u|^{\frac{y}{y-1}}-x\right)^{2}\left(u_{x}\right)^{14} d x d y
\end{aligned}
$$

in $\mathcal{A}^{p}=\left\{u \in W^{1, p}(\Omega ; R): u(0, \cdot)=0, u(1, \cdot)=1\right\}$, where $1 \leqslant p \leqslant+\infty$ and $\Omega=(0,1) \times\left(\frac{3}{2}, \frac{5}{2}\right)$. Foss showed [10] that the problem has an amazing property that the infimum of $I(\cdot)$ in $\mathcal{A}^{p}$ depends continuously on the Sobolev exponent $p$, more precisely

$$
\inf _{u \in \mathcal{A}^{p}} I(u)= \begin{cases}0, & 1 \leqslant p<\frac{3}{2} \\ p-\frac{3}{2}, & \frac{3}{2} \leqslant p<\frac{5}{2} \\ 1, & \frac{5}{2} \leqslant p\end{cases}
$$

furthermore he showed that for $1 \leqslant p<\frac{3}{2}$, the minimizer of $I(u)$ in $\mathcal{A}^{p}$ is given by $\hat{u}(x, y)=x^{\frac{y-1}{y}}$, while for $p \geqslant \frac{3}{2}$, the infimum of the problem is unattainable. 
Moreover, minimizing sequences were analytically constructed [10] for $p \geqslant \frac{3}{2}$, which coincide with $\hat{u}(x, y)=x^{\frac{y-1}{y}}$ on the sub-domain $(0,1) \times\left(p, \frac{5}{2}\right)$.

Given $L_{M}$ and $N_{M}$, let a rectangular mesh be given by

$$
x_{i}^{M}=\frac{i}{L_{M}}, \quad i=0,1, \ldots, L_{M}, \text { and } y_{j}^{M}=\frac{3}{2}+\frac{j}{N_{M}}, j=0,1, \ldots, N_{M},
$$

and let $\mathcal{T}^{h_{M}}$ be the triangulation of $\Omega$ with $h_{M}=h\left(L_{M}, N_{M}\right)=\frac{\sqrt{L_{M}^{2}+N_{M}^{2}}}{L_{M} N_{M}}$ given by dividing each rectangle into an upper triangle and a lower triangle, which are respectively above and below the diagonal with positive slope. Define

$$
\mathcal{A}_{h_{M}}=\left\{u \in C(\bar{\Omega}) \cap \mathcal{A}^{p}:\left.u\right|_{K} \text { is affine, } \forall K \in \mathcal{T}^{h_{M}}\right\} .
$$

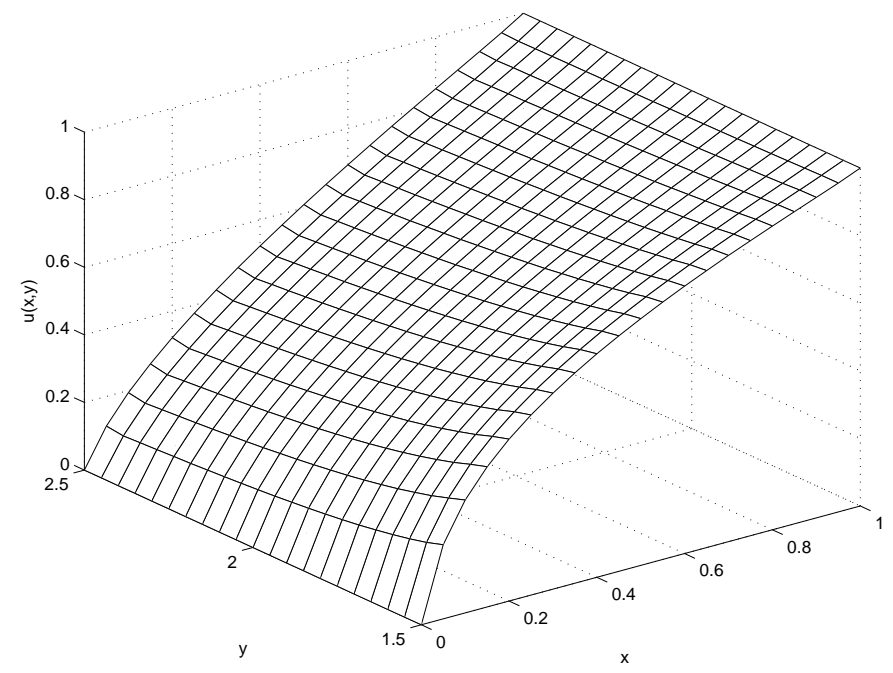

Figure 3. The standard finite element solution on the $20 \times 20$ mesh.

Numerical experiments show that typical numerical solutions obtained by the standard finite element methods have large derivatives near $x=0$ as is shown in Figure 3. Hence, we take the singular set $E=\{0\} \times\left[\frac{3}{2}, \frac{5}{2}\right]$ and $C_{1}\left(h_{M}\right)=$ $C_{2}\left(h_{M}\right)=\frac{\sqrt{2}}{2} h_{M}$, i.e. $\widetilde{\Omega}_{h_{M}}^{E}=\left\{(x, y):(x, y) \in\left[x_{0}^{M}, x_{1}^{M}\right] \times\left[y_{0}^{M}, y_{N_{M}}^{M}\right]\right\}$. Let $I_{M}^{E}(u ; p)$ be given by (3.5) and (3.6) with $\alpha_{h_{M}}(x, y)>\alpha_{1}=10^{-2}$ for all $(x, y) \in \widetilde{\Omega}_{h_{M}}^{E}$. For simplicity, $\alpha_{h_{M}}(x, y)$ is taken to be piece-wise constant. Assume that the leading term of the singularity has the form $\gamma(y) x^{s(y)}$ and evaluate $\gamma\left(y_{j}^{M}\right)>0$ 
and $s\left(y_{j}^{M}\right) \in(0,1)$, for $j=0,1, \ldots, N_{M}$, by the least square method using the nodal values of the numerical solutions $\left\{u_{h_{M}}\left(x_{i}^{M}, y_{j}^{M}\right)\right\}_{i=1}^{3}, j=0,1, \ldots, N_{M}$ near the singular set $E, \alpha_{h_{M}}(K)$ are so taken that the $l^{2}$-error between $u_{h_{M}}$ and $\gamma(y) x^{s(y)}$ on $\left(x_{i}^{M}, y_{j}^{M}\right)$ for $1 \leqslant i \leqslant 3$ and $0 \leqslant j \leqslant N_{M}$ is minimized. To further reduce the complexity, in our computation, we take

$$
\alpha_{h_{M}}(K)= \begin{cases}\bar{\alpha}_{h_{M}}^{1}, & \text { if } K \subset \widetilde{\Omega}_{h_{M}}^{E} \text { is an upper triangle; } \\ \bar{\alpha}_{h_{M}}^{2}, & \text { if } K \subset \widetilde{\Omega}_{h_{M}}^{E} \text { is a lower triangle. }\end{cases}
$$

First, we consider the case when $1 \leqslant p<\frac{3}{2}$. Our numerical experiments show that in such a case $h=h_{M}$ is sufficient to guarantee convergence. Take $p=1.2$. For $L_{1}=N_{1}=20$, the numerical solution $u_{h_{1}}$ produced by the truncation method with $\alpha_{h_{1}}(K) \in\{0.3,2.0\}$ is shown in Figure 4, and the point-wise relative error between the numerical solution $u_{h_{1}}$ and the absolute minimizer $\hat{u}$ is shown in Figure 5. For $L_{2}=N_{2}=40$, the point-wise relative error of the numerical solution $u_{h_{2}}$ produced by the truncation method with $\alpha_{h_{2}}(K) \in\{0.75,2.75\}$ is shown in Figure 6. The convergence behavior of the truncation method for $p=1.2$ is shown in Figure 7.

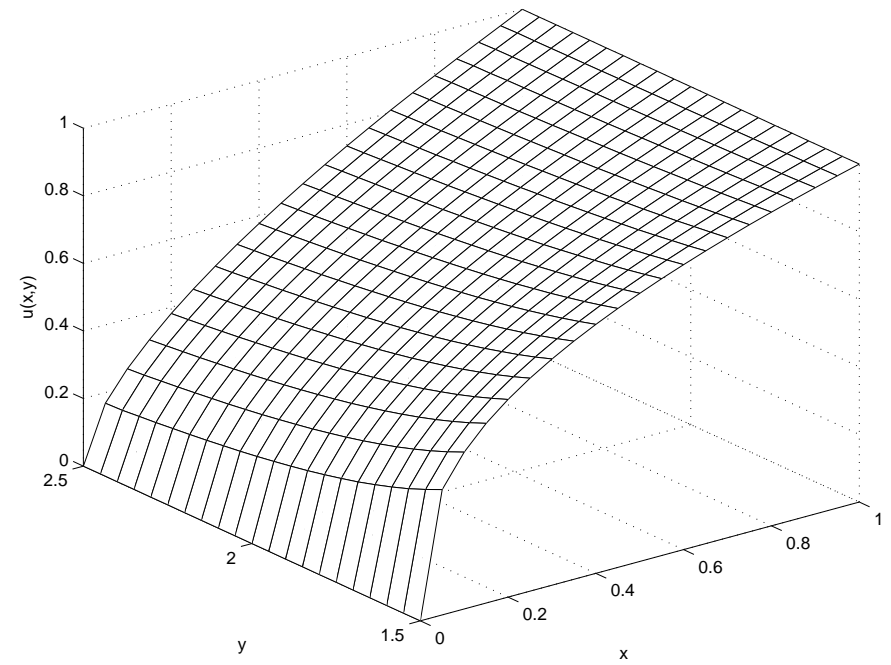

FiguRE 4. The numerical solution produced by the truncation method for $p=1.2$ and $\alpha_{h_{1}}(K) \in\{0.3,2.0\}$ on the $20 \times 20$ mesh. 


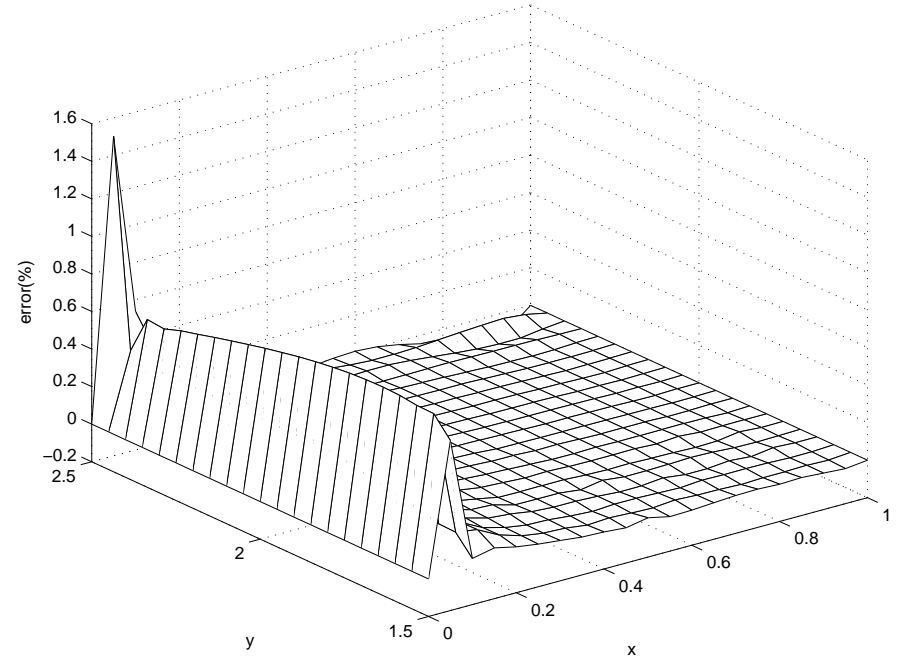

Figure 5. Point-wise relative error for $p=1.2$ and $L_{1}=N_{1}=20$.

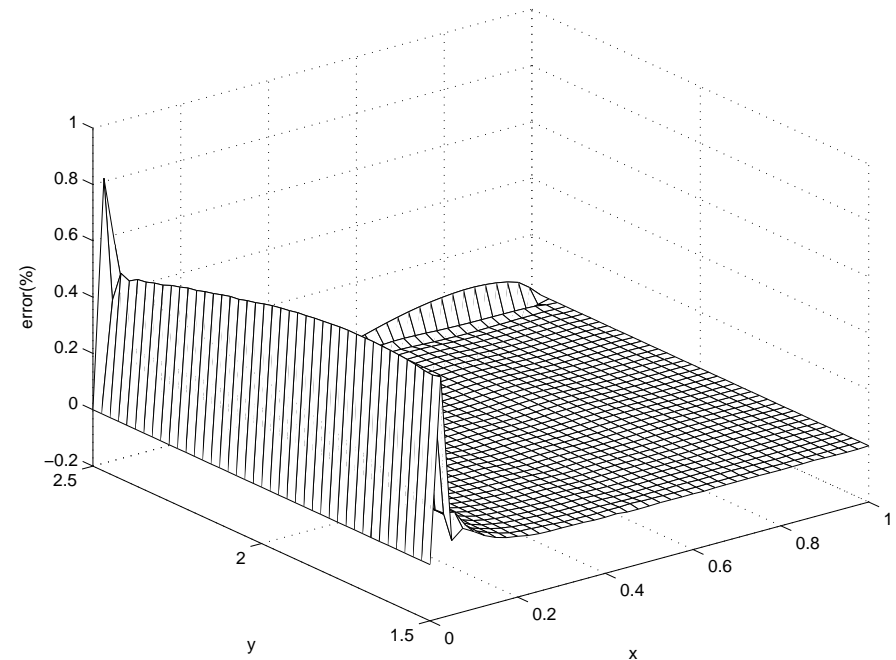

Figure 6. Point-wise relative error for $p=1.2$ and $L_{2}=N_{2}=40$.

Next, we consider the case when $\frac{3}{2} \leqslant p<\frac{5}{2}$. Take $p=2.0$. Recall that in this case the unattainable infimum of $I(\cdot)$ in $\mathcal{A}^{p}$ is $p-\frac{3}{2}=0.5$. Our numerical experiments show that in such a case adaptively refined meshes do produce much better numerical solutions. Starting from the initial $40 \times 40$ uniform mesh $\left(L_{1}=\right.$ $N_{1}=40$ ), the numerical solution $u_{h_{1}}$ produced by the truncation method with 


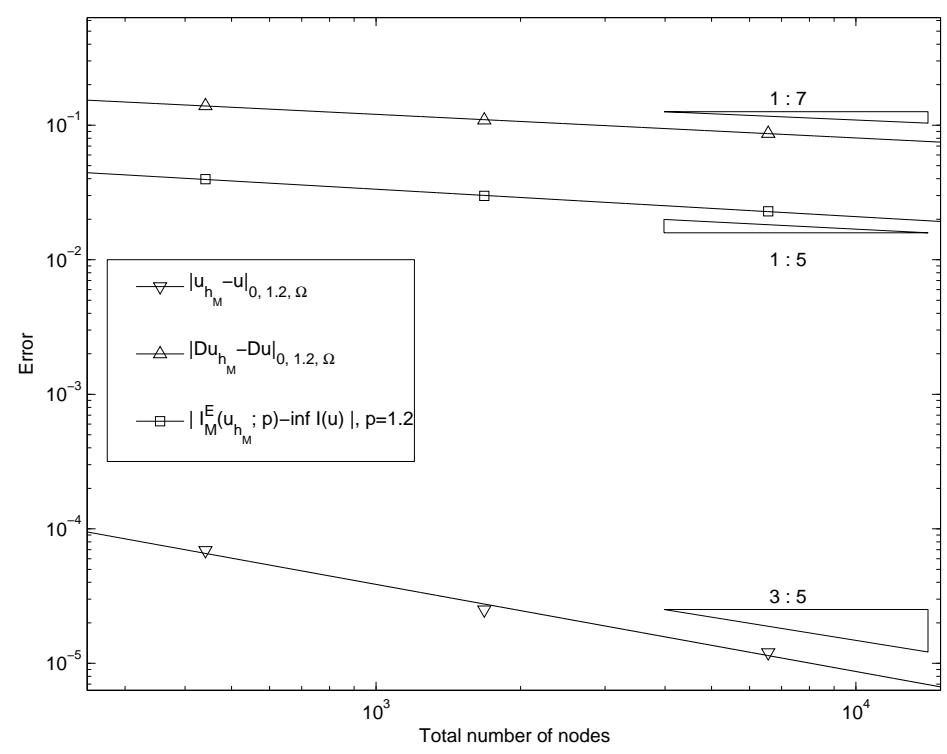

Figure 7 . Convergence rates of the truncation method for $p=1.2$.

$\alpha_{h_{1}}(K) \in\{0.75,2.75\}$ on a refined mesh is shown in Figure 8. As a comparison, a function, which is taken from an analytically constructed minimizing sequence given in [10], is interpolated on the same mesh and shown in Figure 9. Figure 10 shows the convergence rates of $I_{M}^{E}\left(u_{h_{M}} ; p\right)$ to the infimum 0.5 and the $L^{p}$ errors of $\left[u_{h_{M}}-\hat{u}\right]$ and $\left[D u_{h_{M}}-D \hat{u}\right]$ on $y>p$ with $h(K)=h_{M}$ everywhere except on the refined area, where the original mesh is evenly refined in the faster variant direction among $x$ and $y$ by a factor of 10 and $h(K)=\sqrt{\frac{101}{200}} h_{M}$.

Our numerical experiments clearly show that the numerical solutions $u_{h_{M}}$ successfully captured the key features of the minimizing sequence, more precisely, $u_{h_{M}}$ has a sharp variance near the line $y=p=2.0$, the sequence $\left\{u_{h_{M}}\right\}$ converges to $\hat{u}(x, y)=x^{\frac{y-1}{y}}$ on the sub-domain where $y>p=2.0$ and diverges in $W^{1, q}(\Omega)$ for all $q \in[1, \infty]$ (see Figure 11), and hence imply that in this case the infimum is not attainable, at least if the singular set is limited on $x=0$.

Similarly, we can obtain numerical solutions for the case $p \geqslant 2.5$ when the unattainable infimum is 1.0. A typical numerical solution $u_{h_{1}}$ produced by the truncation method on a mesh adaptively refined from the initial $40 \times 40$ uniform mesh $\left(L_{1}=N_{1}=40\right)$ is shown in Figure 12, which again matches well to the corresponding minimizing sequences given in [10]. The convergence behavior of 


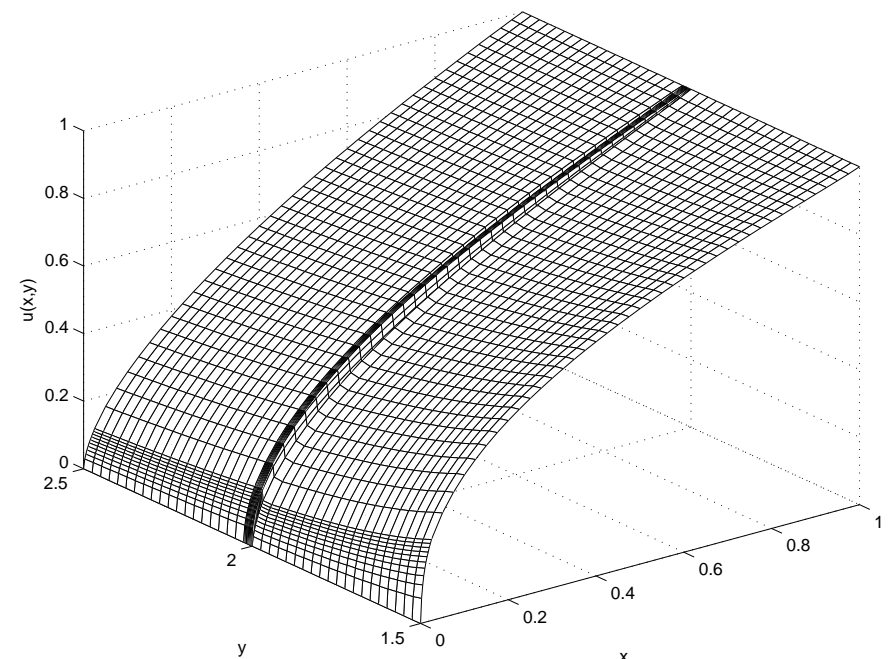

Figure 8. The numerical solution $u_{h_{1}}$ with $\alpha_{h_{1}}(K) \in\{0.75,2.75\}$ for $p=2.0$ on a mesh refined from the $40 \times 40$ uniform one.

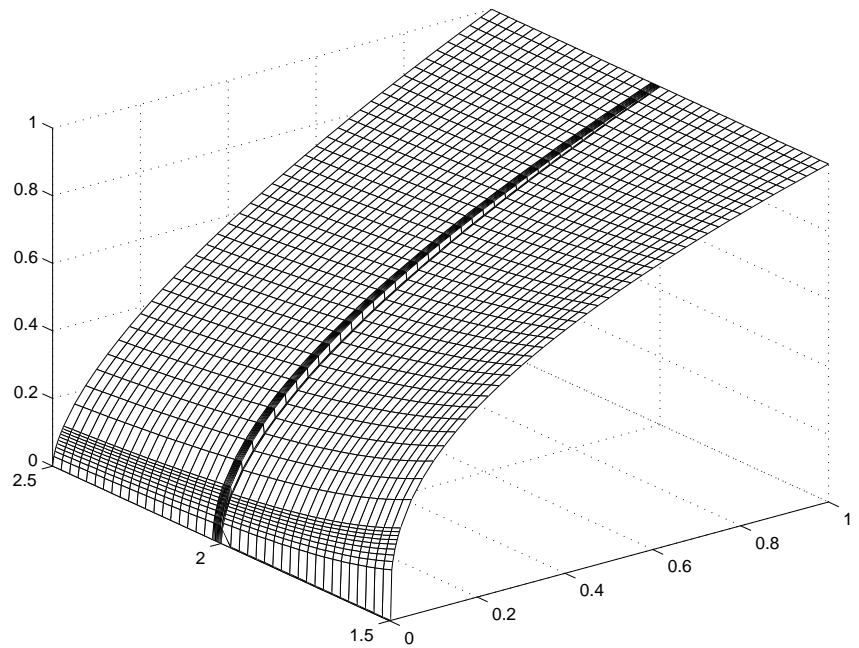

FIGURE 9. The interpolating function of a function taken from an analytically constructed minimizing sequence for $p=2.0$.

$I_{M}^{E}\left(u_{h_{M}} ; p\right)=I\left(u_{h_{M}}\right)$ to the unattainable infimum 1.0 is shown in Figure 10, and the divergence of $\left\{\left\|u_{h_{M}}\right\|_{1, q}\right\}$ for $p \geqslant \frac{5}{2}$ and $q \in[1, \infty]$ is shown in Figure 11 .

Remark 4.1. In the case when $p \geqslant \frac{3}{2}$, even though $\widetilde{\Omega}_{h_{M}}^{E}=\{(x, y):(x, y) \in$ $\left.\left[x_{0}^{M}, x_{1}^{M}\right] \times\left[y_{0}^{M}, y_{M_{M}}^{M}\right]\right\}$, the truncation function takes effect only when $y>p$, since on $\widetilde{\Omega}_{h_{M}}^{E} \cap\{(x, y): y \leqslant p\}$ the $W^{1, p}$-norm of $u_{h_{M}}$ is unbounded. 


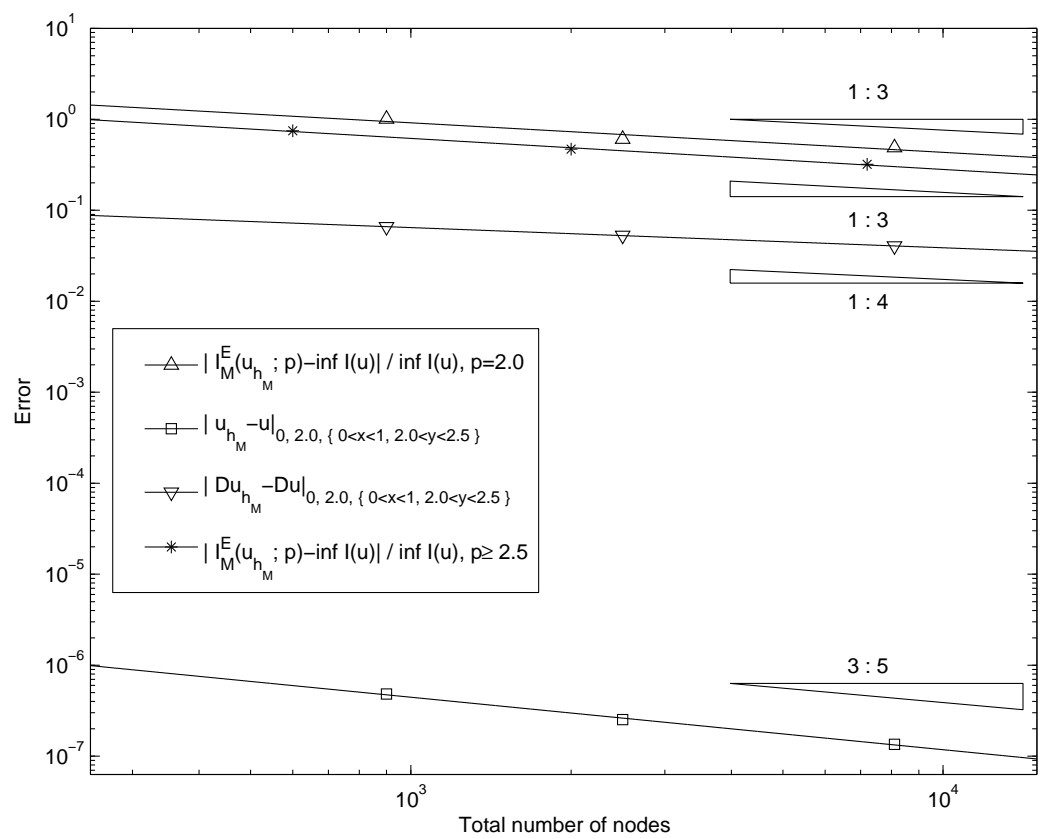

Figure 10. Convergence of $I_{M}^{E}\left(u_{h_{M}} ; p\right)$ to the infimum $p-\frac{3}{2}$ for $p=2.0$ and $p=2.5$ respectively and the $L^{p}$ errors of $u_{h_{M}}$ and $D u_{h_{M}}$ on $y>p$ for $p=2.0$.

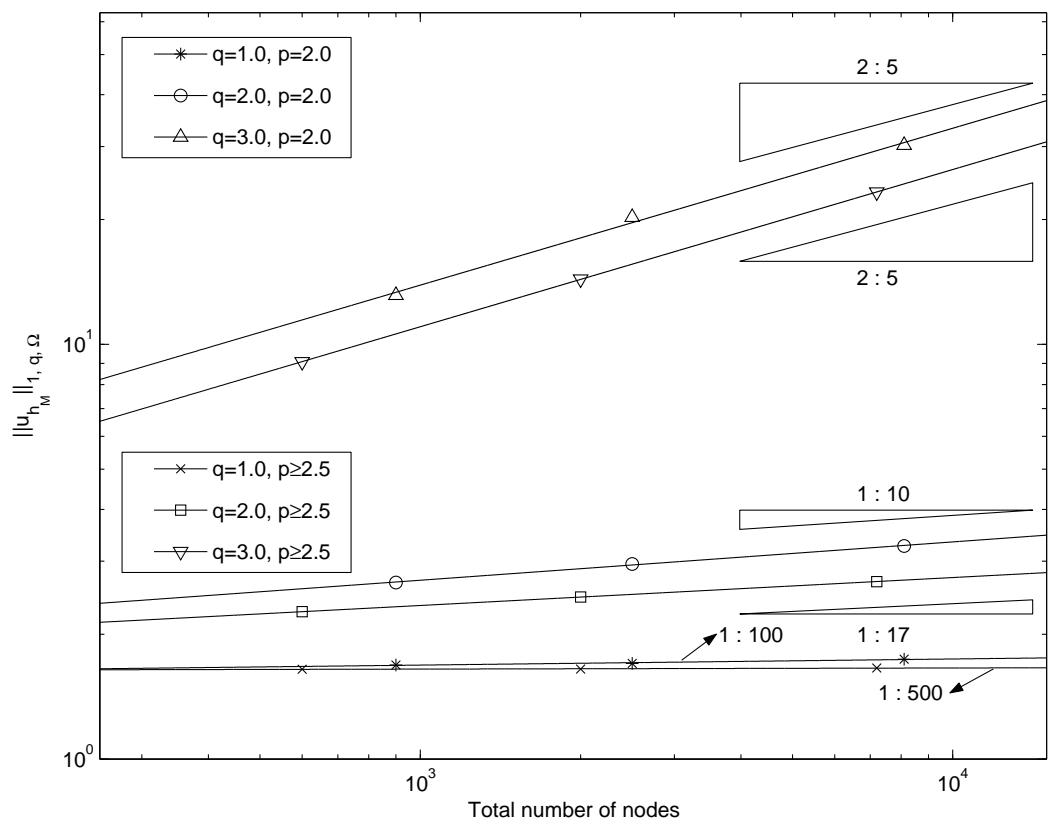

FiguRE 11. Divergence of $\left\{\left\|u_{h_{M}}\right\|_{1, q}\right\}$ for $p \geqslant \frac{3}{2}$ and $q=1.0,2.0,3.0$. 


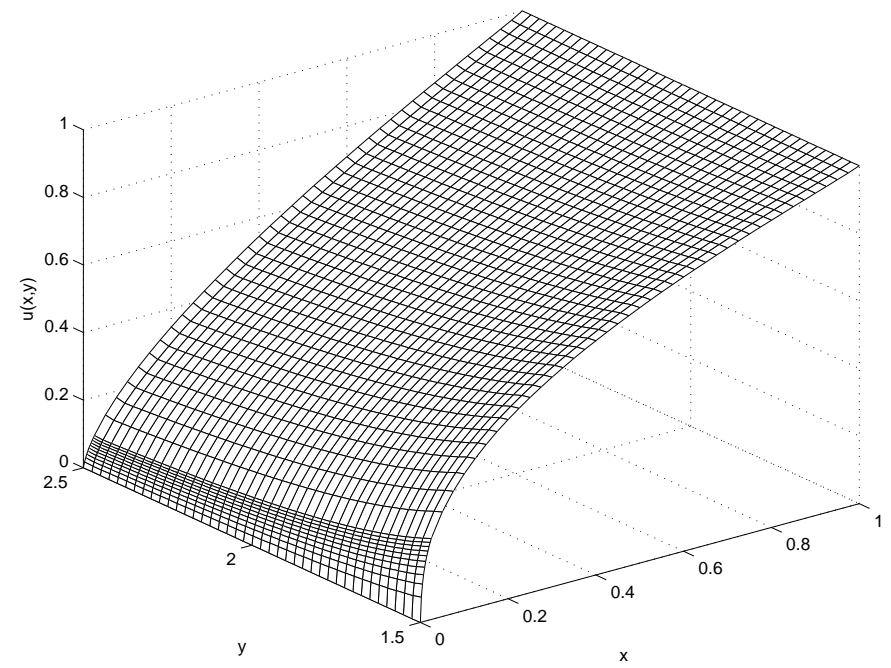

FiguRE 12. A typical numerical solution for $p \geqslant 2.5$.

Remark 4.2. We did not discuss the effect of numerical quadrature which can be crucial in the computation of singular minimizers. In our computation, for the 1-dimensional problem we used three-point Gaussian quadrature formula and for the 2-dimensional problem we used seven-point integration rule, which turned out to be accurate enough. Our numerical experiments show that, for the 2dimensional problem, if only the three nodal points are used in the numerical quadrature the algorithm fails to produce convergent numerical results.

\section{REFERENCES}

[1] R. A. Adams, Sobolev Spaces, Academic Press, New York, 1975.

[2] J. M. Ball and V. J. Mizel, Singular minimizers for regular one dimensional problems in the calculus of variations, Bull. Am. Math. Soc. 11(1984), 143-146.

[3] J. M. Ball and V. J. Mizel, One-dimensional variational problems whose minimizers do not satisfy the Euler-Lagrange equation, Arch. Rational Mech. Anal. 90(1985), 325-388.

[4] J. M. Ball and G. Knowles, A numerical method for detecting singular minimizers, Numer. Math. 51(1987), 181-197.

[5] J. M. Ball, The Calculus of Variations and Material Sciences, Quarterly of Applied Mathematics, 56(1998), 719-740.

[6] P. G. Ciarlet, The Finite Element Method for Elliptic Problems, North HollandAmsterdam, 1978.

[7] B. Dacorogna, Direct Methods in the Calculus of Variations, Apllied Math. Sciences, vol. 78, Springer Verlag, 1989. 
[8] A. M. Davie, Singular minimizers in the calculus of variations in one dimension, Arch. Rational Mech. Anal. 101(1988), 161-177.

[9] R. E. Edwards, Functional Analysis, Holt, Rinehart and Winston, 1965.

[10] M. Foss, Examples of the Lavrentiev phenomenon with continuous Sobolev exponent dependence, Journal of Convex Analysis 10(2)(2003), 445-464.

[11] S. Lang, Real and Functional Analysis, Springer-Verlag, New York, 1993.

[12] M. Lavrentiev, Sur quelques problèmes du calcul des variations. Ann. Math. Pure Appl. 4(1926), 7-18.

[13] Z.- P. Li, Element removal method for singular minimizers in variational problems involving the Lavrentiev phenomenon, Proc. Roy. Soc. Lond., 439A(1992), 131-137.

[14] Z.-P. Li, A numerical method for computing singular minimizers, Numer. Math. 71(1995), 317-330.

[15] Z.-P. Li, Element removal method for singular minimizers in hyperelasticity, Math. Models Methods Appli. Sci., 5(1995), 387-399.

[16] Z.-P. Li, A theorem on lower semicontinuity of integral functionals, Proc. Roy. Soc. Edinb., 126A(1996), 363-374.

[17] B. Manià, Soppa un esempio di Lavrentieff, Ball. Unione Mat. Ital. 13(1934), 147-153. 